\title{
Prevention is better than cure: Understanding metabolic syndrome (MetS) and the occupational risks for perioperative nurses
}

Follow this and additional works at: https://www.journal.acorn.org.au/jpn

Part of the Perioperative, Operating Room and Surgical Nursing Commons c) (i)

This work is licensed under a Creative Commons Attribution 4.0 License.

\section{Recommended Citation}

Turnbull, Daniel and Foran, Paula (2021) "Prevention is better than cure: Understanding metabolic syndrome (MetS) and the occupational risks for perioperative nurses," Journal of Perioperative Nursing: Vol. 34 : Iss. 3 , Article 7.

Available at: https://doi.org/10.26550/2209-1092.1149

https://www.journal.acorn.org.au/jpn/vol34/iss3/7

This Discussion paper is brought to you for free and open access by Journal of Perioperative Nursing. It has been accepted for inclusion in Journal of Perioperative Nursing by an authorized editor of Journal of Perioperative Nursing. 


\section{Prevention is better than cure: Understanding metabolic syndrome (MetS) and the occupational risks for perioperative nurses}

\section{Cover Page Footnote}

Metabolic syndrome (MetS) is a condition with interconnected abnormalities of the metabolic system that has been labelled by the World Health Organization as a main cause of death worldwide. Risk factors for MetS include occupational stress, disturbances to circadian rhythms, sleep disorders and changed eating habits, which are all associated with shift work. As shift work and occupational stress are common in perioperative nursing, the risk of developing MetS is increased for perioperative nurses. This discussion paper aims to bring an awareness and understanding of MetS to perioperative nurses and identifies the occupational risks in the perioperative environment that may lead to its development. It also presents some possible strategies to mitigate the risk factors or prevent this condition for perioperative nurses in the future. 


\section{Authors}

Daniel Turnbull

GradDipNsg (Periop), BN, RN MACN

Dr Paula Foran

PhD, RN, FACORN, FACPAN, MACN

\title{
Prevention is better than cure: Understanding metabolic syndrome (MetS) and the occupational risks for perioperative nurses
}

\begin{abstract}
Metabolic syndrome (MetS) is a condition with interconnected abnormalities of the metabolic system that has been labelled by the World Health Organization as a main cause of death worldwide. Risk factors for MetS include occupational stress, disturbances to circadian rhythms, sleep disorders and changed eating habits, which are all associated with shift work. As shift work and occupational stress are common in perioperative nursing, the risk of developing MetS is increased for perioperative nurses. This discussion paper aims to bring an awareness and understanding of MetS to perioperative nurses and identifies the occupational risks in the perioperative environment that may lead to its development. It also presents some possible strategies to mitigate the risk factors or prevent this condition for perioperative nurses in the future.
\end{abstract}

Keywords: metabolic syndrome, circadian misalignment, shift work, occupational stress

\section{Introduction}

Metabolic syndrome (Mets) was previously known as 'syndrome $x^{\prime}$ and was first recognised by Gerald M Raevan in the 1980's'. The pathophysiology of MetS is complex and comprises interconnected abnormalities of the metabolic system including lipid and glucose metabolism. MetS is diagnosed by the simultaneous presence of three or more of the following factors: hypertension, dyslipidaemia, central obesity and hypertriglyceridemia ${ }^{1-4}$. The syndrome has been linked to co-morbidities of the liver and reproductive system, thrombotic states and inflammatory diseases. It has been identified as having an increased risk of mortality, with the World Health Organization labelling it as a main cause of death worldwide alongside cardiovascular disease, type 2 diabetes mellitus and breast cancer ${ }^{1,5,6,7}$. Research has also theorised that MetS affects approximately 30 per cent of the adult population world-wide ${ }^{8}$.

A systematic review of literature by Ranasinghe et al. in 2017 provided an alternative estimate of the prevalence of MetS, estimating that 20 to 25 per cent of the adult population may be suffering from the disorder'. This review was the first to complete a comprehensive systematic evaluation of literature regarding prevalence of MetS in the Asia-Pacific region; however, from a possible 51 countries, only 15 studies were found revealing the importance of further data collection?. In Ranasinghe's study several variables were considered, including age, occupation and gender'. Females generally had a higher prevalence of MetS except in some specific areas, one of which was rural Australia?. 
More recent studies found similar findings where MetS had an increased prevalence in women; however, data around prevalence in shift workers varied from nine per cent to 30 per cent, with some studies suggesting a two per cent prevalence in nurses specifically ${ }^{3,6,8,9}$. While most studies found age to be a factor in prevalence - the under 40 age group were at high risk - other studies acknowledged the presence of MetS in all age groups $3,4,6,9$. Despite the variation in data and research on the prevalence of MetS, which may be due to different study designs, it is important for the health of all nurses to investigate the occupational risks of shift work and the potential to develop MetS with its associated risk of mortality ${ }^{10,11}$.

\section{MetS and periperative nursing}

Perioperative nursing often involves shift work, being on-call and changing rosters. Meal breaks can be short, requiring nurses to alter their dietary habits and eat quickly while at work. Perioperative nurses may experience occupational stress with long work hours and physically demanding tasks such as lifting heavy instrument trays, moving and positioning patients and wearing lead aprons. Thus there are a number of occupational risks for MetS associated with working in the operating room.

\section{Shift work}

Shift work has been identified as increasing the risk of developing MetS $3,4,6,10,12,13$. A systematic review and meta-analysis by Wang et al. looked at the risk of MetS associated with shift work. The review of 36 studies, with a combined total of 216527 participants, revealed that shift workers had an increased risk of developing MetS compared to regular day workers $(O R=1.35,95 \% \mathrm{Cl}$ : $1.24-1.48 ; 12=74.6 \%)^{12}$. Wang et al. also identified a further increase of risk in rotating shift workers ${ }^{12}$, and this was also highlighted by Khosravipour et al. in their similar systematic review of 38 observational studies ${ }^{10}$. The increased risk of developing MetS in shift workers has been attributed to the desynchronisation, or misalignment, of the circadian rhythm and workers' disrupted sleepwake cycles s,6,7, $^{5}$.

\section{Circadian misalignment}

Circadian rhythm influences temperature and the sleep-wake cycle and is synchronised by the retina's exposure to light which stimulates photosensitive cells connected to the suprachiasmatic nucleus in the hypothalamus gland in the brain ${ }^{14,15}$. The suprachiasmatic nucleus then innervates the sympathetic nervous system which regulates humoral, neural and endocrine signals that lead to predictable behaviours of metabolism and physical cellular performance ${ }^{14,15}$. Extended exposure to light disrupts this circadian rhythm, especially in night shift workers, and may result in insulin resistance and glucose intolerance from altered hormonal secretions, potentially leading to the development of MetS 3,14 . A systematic review of metabolic and cardiovascular consequences of shift work by Kervezee et al. identified evidence that disruption to circadian rhythms, or circadian misalignment, is linked to elevated glucose levels and insulin resistance in varying degrees, particularly in the majority of night shift workers ${ }^{14}$. The review also found that shift workers were at a greater risk of developing metabolic disorders from short-term circadian rhythm misalignment ${ }^{14}$. The risk of developing MetS from circadian misalignment is compounded by disrupted sleep and altered eating regimes both of which are common with shift work.

\section{Sleep deprivation and sleep disorders}

Sleep deprivation has been theorised to affect the endoplasmic reticulum within cellular structures. These organelles are responsible for processing secretory and membrane proteins which have a link to insulin functionality, lipidystrophy, obesity and type 2 diabetes? Shift work, quick turn-around times between shifts, duration of shifts and night shift may all contribute to the development of sleep disorders, which are known risk factors for MetS ${ }^{6,7,15}$. A small cross-sectional study on MetS in night shift workers $(n=60)$, revealed that insomnia symptoms were found in 40 per cent of the participants?. Rosa et al. also identified in their systematic review of randomised control trials and observation studies $(n=24)$ of shift work and nurses' health, that sleep disorders were more prevalent for staff rotating onto morning shift from night shifts when following a $3 \times 8$-hour rotation schedule ${ }^{6}$. Other studies have suggested night shift workers sleep less than day workers, and some studies show workers on a rapidly changing shift rotation sleep fewer hours than workers permanently on night shift ${ }^{6,14}$. Rosa et al. also noted that all shift-work hours interfere with at least one main meal and that appetite levels are affected by short sleep cycles ${ }^{6}$

\section{Changes in eating regime and appetite}

Increased risk of MetS may come from appetite changes in shift workers due to unregulated meal times, sleep disturbances, changes in lifestyle and demands of shift work ${ }^{6}$. Changes to eating regimes can lead to altered insulin responses, and disrupted secretion of appetite hormones may lead to higher caloric consumption ${ }^{6,14}$. A small study by Molzof et al. comparing female 
day- and night-shift workers ( $n=17)$

and the impact of meal timing

on cardiometabolic syndrome indicators, revealed that irregular eating patterns were observed more in shift workers, and that night shift workers' food intake was associated with multiple risk factors specific for MetS ${ }^{5}$. This study also found that increased lipid levels and weight gain was associated with increased food consumption in the evening 5 . While the research by Molzof et al. had a small sample size and was restricted to female shift workers, other studies have also shown that high calorie consumption by shift workers and calorie consumption in the evening increases the risk of developing MetS $^{2,7}$. Another interesting finding, from a cross-sectional data analysis of nurses ( $n=1638$ ) by Jung et al., is that faster eating speeds have been associated with weight gain and MetS$^{2}$. Jung et al. also found that nurses have meal breaks, on average, between six and 28 minutes long². Higher caloric consumption by nurses during these meal breaks could also be attributed to occupational stressors $^{16}$.

\section{Occupational stress}

Research has shown that occupational stress may increase the risk of MetS $^{8}$. Increased stress has been associated with shift work, specifically rotating shift work, with research theorising that one in every four nurses are being affected ${ }^{6}$. In addition, the perioperative environment can be a stressful environment. Excess stress during work can increase the production of cortisol which could potentially lead to insulin resistance and development of MetS ${ }^{17,18}$. Cortisol production by the adrenal gland is stimulated by adrenocorticotropic hormone from the hypothalamicpituitary-adrenal axis in times of stress, leading to increased energy from gluconeogenesis and lipid mobilisation in adipose tissue ${ }^{7,17}$. A cross-sectional analysis of a multicentre cohort of civil servants $(n=15105)$ by Santos et al. observed that higher levels of cortisol were noted in irregular shift workers on their rostered days off, thereby resulting in prolonged stress responses ${ }^{13}$. Although this analysis did not focus on the nursing profession, the analysis revealed that shift work contributed independently to the risk of developing MetS ${ }^{13}$. Chronic stress can also cause burnout with some studies suggesting a link between burnout and MetS ${ }^{17}$. Others studies suggest there has been no definite association between MetS and burnout, although association with components of MetS were found $^{18}$. Due to the inconsistency of evidence from multiple studies, more research is required on stress-related burnout and MetS $^{18}$.

\section{Recommendations}

Managers need to have an awareness of the risk factors for MetS and put strategies in place to mitigate the occupational risks that could result in nurses developing MetS $^{3,6,13}$. Strategies include raising awareness of MetS, education about diet and the importance of physical exercise in avoiding metabolic disorders, and promotion of healthy behaviours ${ }^{2,3,6}$. Benefit could be gained by introducing a health care program for staff with increased risk of developing MetS and implementing a food diary to help control calorie consumption 2,16. It is known that perioperative nurses suffer fatigue due to emotionally and physically demanding work and staffing practices ${ }^{19}$. Thus, managers also need to critically think about shift scheduling and rotations, and frequency and length of shifts, as well as ensuring adequate rest periods to reduce detrimental effects on staff and increase wellbeing ${ }^{6,15}$.

As the risk of developing MetS is ever present in the perioperative environment, it is important for organisations to screen workers who are exposed to shift work, identify those at high risk of developing this dangerous disorder and provide education and resources to reduce the risk.

Finally, as research into MetS in the perioperative domain is limited, it is recommended that organisations facilitate research in operating suites across Australia to explore the prevalence of and prevention strategies for this potentially dangerous disorder to improve the health of nurses ${ }^{6}$.

\section{Conclusion}

MetS is a complex and potentially fatal condition believed to affect nearly a third of the world's population. Shift work and the associated disturbances to circadian rhythms, sleep and eating habits, as well as occupational stress, are recognised as risk factors for developing MetS and are occupational risks for perioperative nurses. Further research into and reporting about MetS will improve the understanding of the physiological mechanisms underlying MetS and the risk factors that contribute to its development. Increasing awareness among the perioperative nursing profession will enable the development and implementation of strategies that may mitigate the risks factors of MetS and reduce its prevalence thus improving the overall health of the perioperative nursing workforce. 


\section{Acknowledgment}

This paper was submitted to the University of Tasmania as part fulfilment of subject CNA803, Advanced Clinical Nursing Practice, for the Master of Clinical Nursing (Perioperative Nursing). The author sincerely wishes to thank Dr Paula Foran, unit coordinator, for her guidance throughout the master course and work in preparing this paper for publication.

\section{References}

1. Ranasinghe $P$, Mathangasinghe $Y$, Jayawardena R, Hills A, Mirsa A. Prevalence and trends of metabolic syndrome among adults in the Asia-Pacific region: A systematic review. BioMed Central 2017:17(101):1-9.

2. Jung $H$, Dan $H$, Pang $Y$, Kim B, Jeong $H$, Lee JE et al. Association between dietary habits, shift work and the metabolic syndrome: The Korea nurses' health study. Int J Environ Res Public Health 2020;17(20).

3. Nikpour M, Tirgar A, Hajiahmadi M, Hosseini A, Heidari B, Ghaffari F et al. Shift work and metabolic syndrome: A multi-center crosssectional study on females of reproductive age. Biomedical Reports 2019;10(5):311-317.

4. Rosa D, Villa G, Montanari E, Destrebecq A, Terzoni S. The relationship between urolithiasis, metabolic syndrome and nurse shift work: A literature review. Int J Urol Nurs 2020;14(2):57-66
5. Molzof HE, Wirth MD, Burch JB, Shivappa N Hebert JR, Johnson RL et al. The impact of meal timing on cardiometabolic syndrome indicators in shift workers. Chronobiol Int 2017;34(3):337-348.

6. Rosa D, Terzoni S, Dellafiore F, Destrebecq A. Systematic review of shift work and nurses' health. Occup Med 2019;69(4):237-243.

7. Ferraz-Bannitz R, Beraldo RA, Coelho PO, Moreira AC, Castro M, Foss-Freitas MC. Circadian misalignment induced by chronic night shift work promotes endoplasmic reticulum stress activation impacting directly on human metabolism. Biology 2021;10(197):1-13.

8. Kuo W, Bratzke LC, Oakley LD, Kuo F, Wang $\mathrm{H}$, Brown RL. The association between psychological stress and metabolic syndrome: A systematic review and metaanalysis. Obes Rev 2019;20(11):1651-1664.

9. Holanda NCP, Castro Moreno CR, Marqueze EC. Metabolic syndrome components: Is there a difference according to exposure to night work? Chronobiol Int 2018;35(6):801810.

10. Khosravipour M, Khanlari P, Khazaie S, Khosravipour $\mathrm{H}$, Khazaie $\mathrm{H}$. A systematic review and meta-analysis of the association between shift work and metabolic syndrome: The roles of sleep, gender,and type of shift work. Sleep Med Rev 2021;57(101427):1-14. DOI: 10.1016/j. smrv.2021.101427.

11. Loef B, Baarle D, van der Beek AJ, Beekhof PK, van Kerkhof LW, Proper KI. The association between exposure to different aspects of shift work and metabolic risk factors in health care workers, and the role of chronotype. Plos One 2019;14(2):1-14.
12. Wang $Y$, Yu L, Gao $Y$, Jiang L, Yuan L, Wang $P$ et al. Association between shift work or long working hours with metabolic syndrome: A systematic review and dose-response meta-analysis of observational studies. Chronobiol Int 2021;38(3):318-333.

13. Santos AE, Araújo LF, Griep RH, Castro Moreno CR, Chor D, Barreto SM et al. Shift work, job strain, and metabolic syndrome: Cross-sectional analysis of ELSA-Brasil. Am J Ind Med 2018;61(11):911-918.

14. Kervezee L, Kosmadopoulos A, Boivin DB. Metabolic and cardiovascular consequences of shift work: The role of circadian disruption and sleep disturbances. Eur J Neurosci 2020;51(1):396-412.

15. Garde AH, Begtrup L, Bjorvatn B, Bonde JP, Hansen J, Hansen ÅM et al. How to schedule night shift work in order to reduce health and safety risks. Scand J Work Environ Health 2020;46(6):557-569.

16. Bigand T, Cason M, Diede T, Wilson M. Pilot testing an electronic food diary among registered nurses working night shifts. Chronobiol Int 2020;37(9/10):1377-1383.

17. Merces MC, Gomes AMT, Coelho JMF, Servo MLS, Marques SC, Júnior AD. Scientific evidence on the association between burnout and metabolic syndrome: Integrative review. Acta Paul Enferm 2019:32(4):470-476.

18. Chico-Barba G, Jiménez-Limas K, SánchezJiménez B, Sámano R, Rodriguez-Ventura AL, Castillo-Pérez,et al. Burnout and metabolic syndrome in female nurses: An observational study. Int J Environ Res Public Health 2019;16(11):1-11.

19. Nijkamp N, Foran P. The effects of staffing practices on safety and quality of perioperative nursing care - an integrative review. JPN 2021;34(1):e15-e22. 